CMV polyradiculopathy in AIDS-suggestions for new strategies in treatment

Cytomegalovirus (CMV) polyradiculopathy presenting as a cauda equina syndrome is now a well described disorder in AIDS and is estimated to occur in $2 \%$ of AIDS patients with neurological problems.' Although dihydroxypropoxymethylguanine (DHPG, ganciclovir) has been shown to be effective, the optimum dose and duration of induction therapy have not been determined. Trisodium phosphonoformate guanine (foscarnet) has been shown to be efficacious in the treatment of some of the other complications of CMV, such as retinitis, but there have been no reports of its use in the polyradiculopathy syndrome.

A 41 year old bisexual man, HIV seropositive since 1984, progressed to AIDS in August 1988 when he developed Pneumocystis carinii pneumonia. In April 1990 he was admitted with a two week history of increasing weakness and paraesthesia in the legs, urinary frequency and two episodes of faecal incontinence. On examination the lower limbs were hypotonic and weak: MRC grade 4 proximally and grade 3 on the right, grade 1 on the left distally. The knee and ankle jerks were absent and the plantar responses equivocal. There was a mild sensory deficit with reduction to pinprick sensation over the $S_{1}$ dermatome on the left. Anal tone was reduced and the bladder was palpable to the umbilicus. Examination of the upper limbs and cranial nerves was normal. There was no evidence of cytomegalovirus retinitis.

A thoracolumbar myelogram was normal. Cerebrospinal fluid examination showed 44 WBC, predominantly neutrophils, and the protein was $1.7 \mathrm{~g} / \mathrm{l}$. No organisms were cultured. Cytological examination and syphilis serological tests were negative. Nerve conduction tests on the lower limbs were consistent with a lumbar radiculopathy.

A presumptive diagnosis of CMV polyradiculopathy was made and treatment was started with ganciclovir $10 \mathrm{mg} / \mathrm{kg} /$ day, 72 hours after admission. Zidovudine was stopped. Despite three weeks of treatment he continued to deteriorate and developed complete flaccid paralysis of his legs and required a suprapubic urinary catheter in situ. Empirically, foscarnet $(57 \mathrm{mg} / \mathrm{kg} \mathrm{TID}$ ) was substituted for the ganciclovir. Zidovudine was reinstituted initially at a dose of $300 \mathrm{mg}$ gradually increasing to $1000 \mathrm{mg}$ daily.

The first signs of improvement were noted after two weeks of treatment with foscarnet. Over the next five weeks clinical improvement continued. However, owing to deteriorating renal function after 7 weeks of treatment with foscarnet, ganciclovir at a reduced maintenance dose of $3 \mathrm{mg} / \mathrm{kg} /$ day ( 5 out of 7 days) was reinstituted. This has continued to be self administered at home via a Hickman line. Zidovudine had been discontinued 4 weeks after it was restarted because of anaemia.

Five months after the initial presentation, the patient is mobile around his home using crutches but still requires an indwelling urinary catheter. The most prominent deficit is a left foot drop and absent ankle reflexes.

We report this case to highlight four issues regarding treatment of CMV polyradiculopathy in AIDS patients: firstly, substantial improvement is possible in this condition. Our patient improved from having a complete flaccid paralysis to being mobile with crutches. Secondly, unless the patient's general condition dictates otherwise, a deterioration in spite of treatment should not be a deterrent to the continuation of anti CMV therapy. With regards to drug dosages and the necessity for maintenance treatment, much of the experience from CMV retinitis has been extrapolated to CMV polyradiculopathy. A review of the literature shows that induction therapies of ganciclovir $5-10 \mathrm{mg} / \mathrm{kg} /$ day for between 10 to 21 days are being used. This case suggests that high dose induction therapy of anti-CMV treatment should be continued at least until there is no further neurological improvement. Finally, although it is difficult to attribute improvement in our patient specifically to foscarnet, this may be an effective alternative to ganciclovir in CMV polyradiculopathy as in, for example, CMV retinitis. Furthermore, as foscarent is less myelotoxic than ganciclovir, zidovudine therapy may be continued. Foscarent should be considered as alternative therapy in cases where no improvement has occurred after two to three weeks of treatment with ganciclovir or in cases intolerant of ganciclovir. Clearly, further studies comparing the efficacy of these two therapies are required.

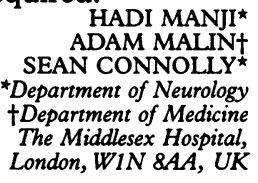

1 De Gans J, Portegies P. Neurological complications of infection with the Human Immunodeficiency Virus type 1. A review. Clin Neurol Neurosurg 1989;91:197-217.

\section{National STD trends in Zambia: 1987-89}

We have previously reported a rise in the number of cases of sexually transmitted diseases (STDs) in Zambia from 1983 to 1987 and expressed concern over the exponential increase in cases of chancroid and syphilis in 